\title{
COMBATING NEAR FAR EFFECTS OF LINEAR MMSE MULTIUSER DETECTION IN CODED OFDM- CDMA
}

\author{
Martin Feuersänger and Volker Kühn \\ University of Bremen, FB-1, Department of Communications Engineering, \\ P.O. Box 3304 40, D-28334 Bremen, Germany, \\ Fax: $+49-421 / 218-3341$ \\ http://www.comm.uni-bremen.de \\ \{feuersaenger,kuehn\}@comm.uni-bremen.de
}

\begin{abstract}
This paper deals with near far effects encountered in MMSE linear multiuser detection and proposes a method to mitigate the transmit-power dependent userspecific bit error rates by introducing an additional postprocessing by a combination of nonlinear parallel and successive interference cancellation. Throughout the paper we consider a quasisynchronous (i.e. asynchronous with coarse synchronization at the receiver) OFDM-CDMA uplink transmission as well as perfectly known channel impulse responses.
\end{abstract}

Keywords: OFDM-CDMA, multiuser detection, interference cancellation, near far problem

\section{Introduction}

In recent years CDMA has been chosen as multiple access technique in various major modern communication systems $[1,2,3,4]$. In this paper we investigate a multi-carrier CDMA (MC-CDMA) system [5, 6] in an uplink situation. As multi-carrier technique we use Orthogonal Frequency Devision Multiplex (OFDM). By using OFDM each subcarrier is only affected by flat fading and the equalization effort is reduced to one tap for each subcarrier.

Since we investigate an asynchronous uplink situation multiuser interference (MUI) can not be effectively suppressed by using orthogonal signature waveforms for spreading. This is only suitable in synchronous environments since asynchronous conditions would destroy the orthogonality of such spreading signatures. As an alternative we can use pseudo noise (PN) signature waveforms as spreading sequences and combat MUI by using multiuser detection (MUD). In this paper we investigate the linear MMSE multiuser detector under the influence of users received with varying powerlevels. Since the MMSE detector 
is only asymptotically near far resistant it is desirable to improve the conditions of reception for users with lower reception power. We suggest to postprocess the received signal by a combination of nonlinear parallel and successive interference cancellation (PIC and SIC).

In section 2 we describe the OFDM-CDMA system and the model used to rank the users into different powerlevels, in section 3 we describe the linear MMSE multiuser detection and its application in the transmission system and proposals to combine the linear MUD with a nonlinear interference cancellation (PIC and SIC). Section 4 gives simulation results. Finally, section 5 contains some conclusions.

\section{System Description}

\subsection{Transmission System}

In the transmitter of the OFDM-CDMA system the information bits $\boldsymbol{d}^{(j)}(k)$ for each user (users count from $1 \leq j \leq J$ ) are encoded by a convolutional code (CC) of rate $R_{c}=1 / 2$. The resulting vector $\boldsymbol{b}^{(j)}(k)$ is then spread by $N_{p}=32$ times repetition of each coded bit $b_{i}^{(j)}(k), 1 \leq i \leq n=2$ and a successive multiplication by a user-specific signature $\boldsymbol{c}^{(j)}$ resulting in $\tilde{\boldsymbol{b}}^{(j)}(k)$. As we consider an asynchronous uplink transmission the signatures $\boldsymbol{c}^{(j)}$ are PN sequences. The entire processing gain $G_{p}$ is $N_{p} / R_{c}=64$.

After interleaving over $N_{c}$ chips in the frequency domain the OFDM transmitter transforms $\tilde{\boldsymbol{b}}^{(j)}(k)$ in the time domain. In this paper the number of subcarriers $N_{c}$ corresponds to the processing gain $G_{p}$. This implies that one information bit $\boldsymbol{d}^{(j)}(k)$ inhabits exactly one OFDM symbol. A cyclic prefix of duration $T_{g}$ precedes each OFDM symbol.

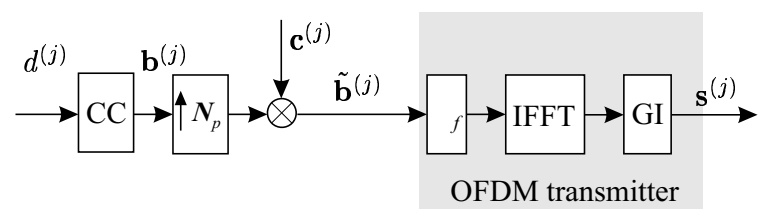

Figure 1. OFDM-CDMA transmitter

The resulting userspecific signals $\boldsymbol{s}^{(j)}(k)$ are then transmitted over $J$ individual $L$-path mobile radio channels. Real and imaginary parts of the channel coefficients $h_{l}^{(j)}(k), 0 \leq l \leq L$ are Gaussian distributed and statistically independent.

At the receiver the cyclic prefix is removed. The received signal is transformed back into the frequency domain by the fast Fourier Transform (FFT). One FFT window transforms all users since we assume a coarse synchronisation (i.e. a 
maximum delay between different users smaller than $T_{g}-\Delta \tau$ where $\Delta \tau$ is the delay spread of the channel. It can be shown that at this stage the convolution with the channel impulse response corresponds to a scalar multiplication of the channel coefficients in the frequency domain $H_{\mu}^{(j)}(k), 0 \leq \mu \leq N_{c}$ with the spread signal $\tilde{b}_{\mu}^{(j)}(k)$ which imposes that each chip is only affected by flat fading.



Figure 2. Single-user OFDM-CDMA receiver

The received vector at the output of the OFDM receiver at time instance $k$ can be expressed by

$$
\boldsymbol{r}(k)=\boldsymbol{A}(k) \boldsymbol{b}(k)+\boldsymbol{n}(k)
$$

where $\boldsymbol{b}(k)$ are the convolutionaly encoded bits $b_{i}^{(j)}(k)$ of all users and $\boldsymbol{A}(k)$ is the system matrix.

The system matrix $\boldsymbol{A}(k)$ is composed by $J$ user specific matrices.

$$
\boldsymbol{A}(k)=\left(\boldsymbol{A}^{(1)}(k) \cdots \boldsymbol{A}^{(J)}(k)\right)
$$

where each of these has the form

$$
\mathbf{A}^{(j)}(k)=\left(\begin{array}{lll}
\mathbf{a}_{1}^{(j)}(k) & & \\
& \ddots & \\
& & \mathbf{a}_{n}^{(j)}(k)
\end{array}\right) .
$$

The Column vectors $\mathbf{a}_{i}^{(j)}(k)=\left(a_{i, 0}^{(j)}(k), \cdots, a_{i, N_{p}-1}^{(j)}(k)\right)^{T}$ contain the elements

$$
a_{i, \nu}^{(j)}(k)=c_{(i-1) N_{p}+\nu}^{(j)} H_{(i-1) N_{p}+\nu}^{(j)}(k), \quad 0 \leq \nu<N_{p}
$$

Those are element-wise products of the signature sequences $\mathbf{c}^{(j)}$ and the channel transfer function $\mathbf{H}^{(j)}(k)$.

The considerations that are necessary to compute the pseudo-inverse $\mathbf{A}^{\dagger}(k)$ are described in [7]. 


\subsection{Power distribution of users}

In order to investigate near far effects in the transmission a model to distribute the different users to different powerlevels is introduced. Hereby concentric rings are assumed around the receiver. The radii are determined by a constant power ratio $\kappa$ in $\mathrm{dB}$ between successive rings and a path loss factor $\mu$ indicating the exponent of the radius. The users are equaly distributed to these powerlevels (constant pdf). We want to emphasize that this leads to a different $E_{b} / N_{0}$ ratio for each powerlevel but results in a constant average over all users. Figure 3 is depicting the constellation of the powerlevels and the resulting user and power distribution as well as the average power for the power scenario considered throughout this paper, i.e a space loss factor of $\mu=3$ and a power ratio of $\kappa=-3 \mathrm{~dB}$. The users are distributed on 4 powerlevels.
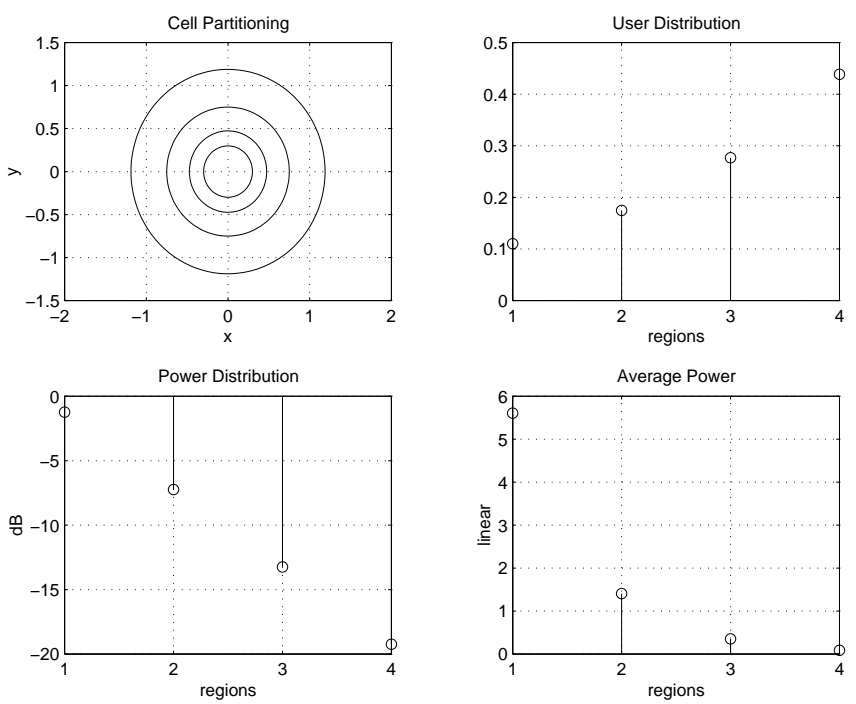

Figure 3. Power distribution for 4 powerlevels $(\mu=3$ and $\kappa=-3 \mathrm{~dB})$

\section{Detection Concepts}

\subsection{Linear Multiuser Detection}

As already mentioned, in linear MUD schemes the pseudo-inverse $\boldsymbol{A}^{\dagger}(k)$ of the system matrix $\boldsymbol{A}(k)$ has to be computed. Thus a linear MUD can be considered as an equalization with knowledge of the structure of the multiple access signal.

In contrast to the zeroforcing decorrelator the MMSE solution of the above problem additionally takes the noise power $\sigma_{N}^{2}$ into account thus reaching a 
compromise between the decorrelation of the interfering users and the avoidance of an amplification of the noise power. In general, MMSE MUD outperforms MUD by the decorrelator [7].

However, in contrast to the decorrelator the MMSE MUD reaches only asymptotically optimal near far resistance ([8],[9]), i.e. for $\sigma_{N}^{2} \rightarrow 0$. This is demonstrated in figure 4. Comparing the bit error rates of the various powerlevels after correcting the $3 \mathrm{~dB}$ differences in $E_{b} / N_{0}$ (refer to section 2.2) the decorrelator shows equal performance for all powerlevels whereas after MMSE MUD lower powerlevels exhibit a weaker performance.

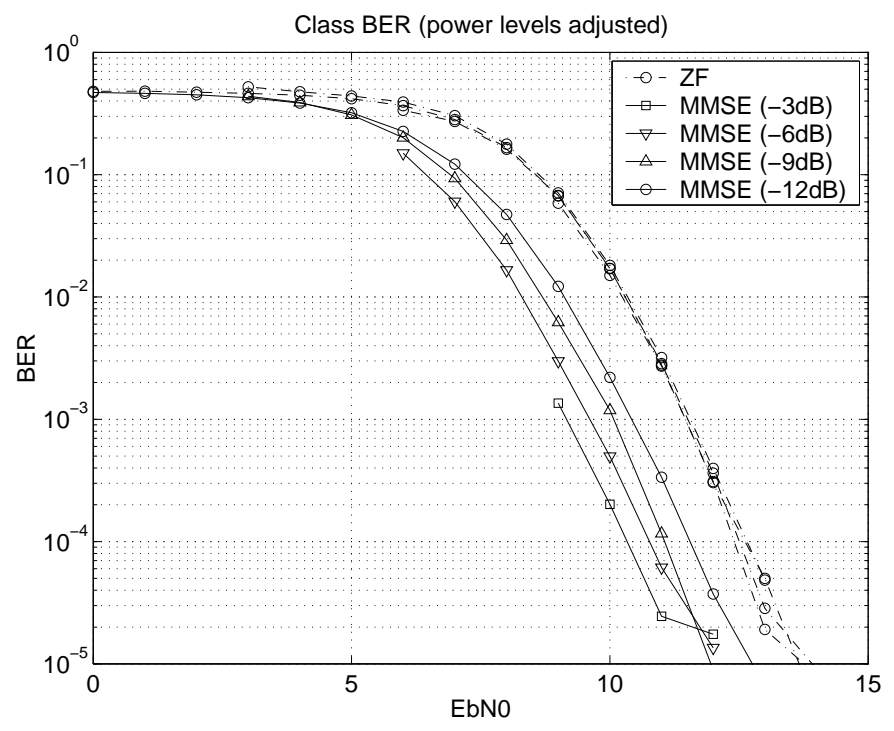

Figure 4. Comparison of decorrelator (ZF) and MMSE MUD in a near far scenario

\subsection{Combating near far effects}

By the nature of the MMSE MUD output and the realizations from [7] we propose a combination of parallel and successive interference cancellation (PIC and SIC) that uses the MMSE output as the initializing signal in order to enhance the signal-to-interference ratio at the input of the FEC decoder. Then, in a multistage process, the highest power users are processed in a PIC loop. The output of the PIC is then transfered to a SIC stage where the reconstructed received signal parts of the users with highest powerlevel are subtracted from the original received signal (before the MMSE MUD) thus reducing the signalto-interference-ratio for the lower power users. The multistage process then steps to the next (lower) powerlevel. This process is depicted in figure 5. 


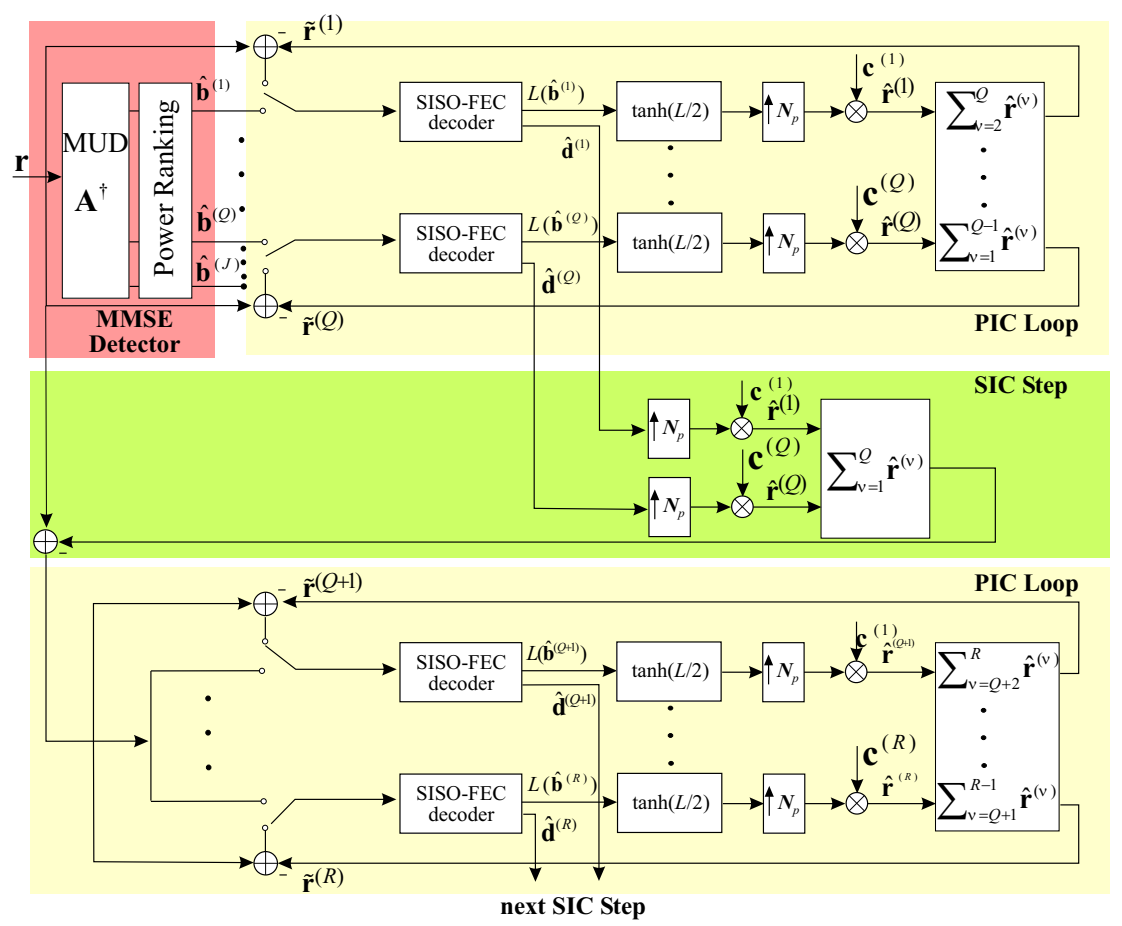

Figure 5. Multistage detector

In this way each of the two nonlinear MUD processes is operating in its predestined environments. Whereas PIC, best operating on users with equal power, is processing the users of one distinct powerlevel, SIC, suitable for conditions with large power variations of the received users, cancels the interference between successive powerlevels.

\section{Performance Analysis}

Simulation results for the proposed multistage MUD are shown in figure 6. The multistage MUD is compared to the MMSE multiuser detection. The different powerlevels are taken into account as described in section 3 .

The multistage MUD is mitigating the near far effects. All powerlevels converge to the bit error performance of the highest powerlevel. Since before detection the users of the lowest powerlevel are freed from all interferers of the higher powerlevels they exhibit the highest performance gain compared to the MMSE MUD. The gain is close to $1 \mathrm{~dB}$. Consequently the users of the highest powerlevel show only little gain by the multistage MUD since they only profit from the PIC Loop within their own powerlevel. 


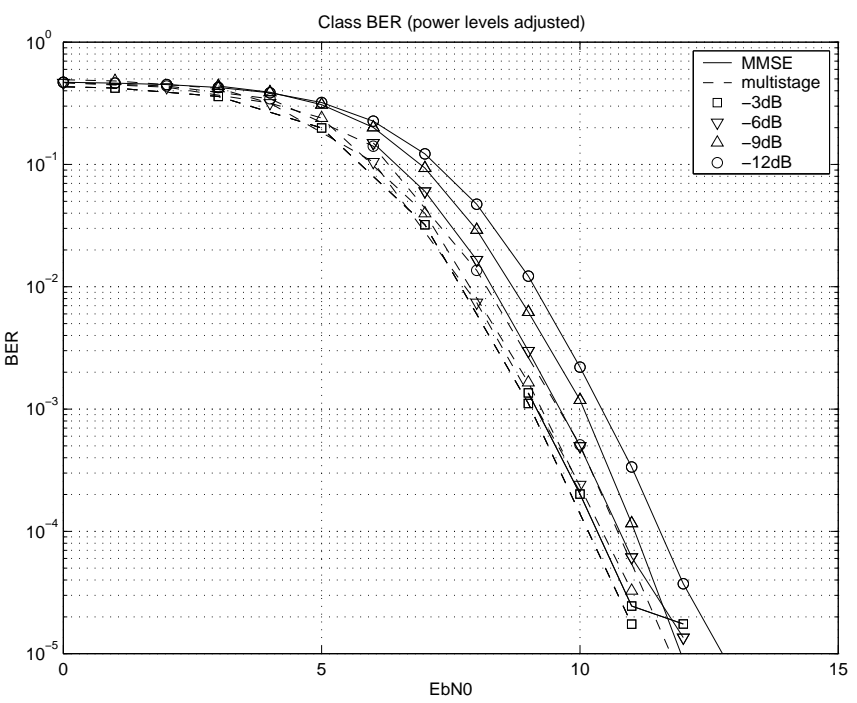

Figure 6. Comparison of MMSE and multistage MUD in a near far scenario

\section{Conclusion}

It is shown that the proposed multistage MUD is indeed leading towards a convergence of the bit error performances of the different powerlevels and thus the near far effects encountered in sole MMSE multiuser detection can be mitigated.

Due to the nature of the system, i. e. the serial connection of parallel and successive interference cancellation in the postprocessing after the MMSE filter, the lowest powerlevel is the most improved with a gain of about $1 \mathrm{~dB}$.

To improve computation effort a proposal would be to additionally investigate a multistage MUD that includes instead of an initial MMSE filter for all users a MMSE filter that aims only at the users of the highest powerlevel. This procedure will reduce computation effort since it involves lower dimension matrix inversions than the multistage MUD process described above. Performance improvement could be achieved by replacing the single user detectors in the system by MMSE filters aiming at the users of the specific powerlevel thus improving the SINR of the following PIC loop.

\section{References}

[1] K. Gilhousen, I. Jacobs, R. Padovani, A.J. Viterbi, L. Weaver, and C. Wheatley, "On the Capacity of a Cellular CDMA System," IEEE Transactions on Vehicular Technology, vol. 40, May 1991. 
[2] E. Dahlman, B. Gudmundson, M. Nilsson, and J. Sköld, "UMTS/IMT-2000 Based on Wideband CDMA," IEEE Communications Magazine, pp. 70-80, September 1998.

[3] T. Ojanperä and R. Prasad, "An Overview of Air Interface Multiple Access for IMT2000/UMTS,” IEEE Communications Magazine, pp. 82-95, September 1998.

[4] A. Toskala, J. Castro, E. Dahlman, M. Latva-Aho, and T. Ojanperä, "Frames FMA2 Wideband-CDMA for UMTS," European Transactions on Communications, vol. 9, no. 4, pp. 325-335, August 1998.

[5] Stefan Kaiser, Multi-Carrier CDMA Mobile Radio Systems - Analysis and Optimization of Detection, Decoding and Channel Estimation, Ph.D. thesis, German Aerospace Center, VDI, January 1998.

[6] A. Dekorsy and K.D. Kammeyer, "A new OFDM-CDMA Uplink Concept with M-ary Orthogonal Modulation," European Transactions on Telecommunications (ETT), vol. 10, no. 4, pp. 377-390, July/August 1999.

[7] V. Kühn, "Combined Linear and Nonlinear Multi-User Detection for Coded OFDMCDMA," in International Symposion of Theoretical Electrical Engineering, Linz, August 2001.

[8] S. Verdu, Multiuser Detection, Cambridge University Press, 1998.

[9] R. Lupas and S. Verdu, "Near-Far Resistance of Multiuser Detectors in Asynchronous Channels," IEEE Transactions on Communications, vol. 38, no. 4, pp. 496-508, April 1990.

[10] K.D. Kammeyer, Nachrichtenübertragung, B.G. Teubner, Stuttgart, second edition, 1996, In German language. 